\title{
Combined Antenna-Channel Characterization for Wireless Communication from Horse Hoof to Base Station
}

\author{
Jasper Goethals*, Günter Vermeeren*, Denys Nikolayev ${ }^{\dagger}$, Margot Deruyck*, Luc Martens*, Wout Joseph* \\ *Department of Information Technology, University of Ghent, IMEC, WAVES, Ghent, Belgium, jasper.goethals@ugent.be \\ †Univ Rennes, CNRS, L'institut d'électronique et de télécommunications de Rennes, UMR 6164, FR-35000 Rennes, France
}

\begin{abstract}
This paper presents a design of an antenna in the complex environment of the horse hoof for sub-gigahertz $(868 \mathrm{MHz})$ communication. The influence of the leg and the ground on the performance were examined by means of finitedifference time-domain simulations. Furthermore, an adaptation was presented to increase the efficiency of the antenna. The first device has a center frequency of $858 \mathrm{MHz}$ and a bandwidth of 99.5 MHz. The total efficiency is $3.12 \%$. Adding the ground to the model results in the ground absorbing most of the radiated power which leads to a total efficiency drop to $1.6 \%$. The center frequency stays the same (at $862 \mathrm{MHz}$ ). The bandwidth decreases to $70 \mathrm{MHz}$. When the horseshoe is connected to the device, the antenna is not tuned anymore, yet the the total efficiency stays $3.08 \%$. This shows that connecting the device to the horseshoe leads to better radiation efficiency. When using LoRa technology, this setup can reach $1631 \mathrm{~m}$ if the hoof is in the air. When the hoof is on the ground, only a range of $115 \mathrm{~m}$ is estimated.
\end{abstract}

Index Terms-channel characterization, modeling, path loss, link budget, horse, LoRA

\section{INTRODUCTION}

Owning a horse is a serious undertaking. The health of this valuable asset is of utmost importance. To monitor its health, one can put a mobile monitoring device on the animal. There already exist products with this function [1],[2], but the performance is not up to the expected standards. The overarching goal of this work is a part of a horse monitoring system. The device should monitor the horse 24/7 and automatically detect anomalies in its behavior while the horse is in its usual environment which include the pasture. This automatic detection of anomalies decreases the response time for a veterinarian intervention which leads to a reduce of the risk of complications and medical costs.

In this paper, the goal is to establish a wireless communication channel between the transmitting monitor device inside the hoof of the horse and off-body hoof antenna to a gateway, e.g. installed in the farm. To cover sufficiently long ranges during training and recreational activities and to be able to directly communicate from the horse to a gateway, radio technologies with suitable propagation characteristics need to be used. Lower frequency waves typically penetrate objects better at lower energy consumption costs, thereby improving the achievable range. However, lower radio frequencies typically require larger antenna sizes, and limit the maximum achievable throughput, thereby limiting device placement options and the amount of monitoring data, respectively [3]. Trade-offs between range, antenna size, and throughput result in using radio frequencies from the $433 \mathrm{MHz}$ ISM (Industrial, Scientific and Medical) bands or the EU $868 \mathrm{MHz}$ SRD (Short Range Device) bands (jointly referred to as sub-GHz bands).

For deciding upon optimal sub-GHz technology and antenna placement, an accurate propagation analysis is required, specifically related to the wireless body area networks (WBAN) propagation characteristics. The physical channel for off-body communication (from the sensor in the hoof to the private/cellular operated gateway) is required. Most research about off-body propagation is focused on humans [4], [5]. However, [6], [7] concluded that radio performance will be significantly affected by an animal's body. Kwong et al. [8] proposed a simple two-path model for off-body communication, but the model has not been validated through measurements. Benaissa et al. [9], [10] investigated off-body path loss and communication for dairy cows in stables at 2.4 $\mathrm{GHz}$ and $868 \mathrm{MHz}$. For on-body communications, the work of Reusens [11] and Roy [5] are relevant for UWB (Ultra Wide Band) and $2.4 \mathrm{GHz}$ frequencies.

With respect to the impact of metal in the channel (horsehoe near the antenna), Tanghe et al. [12] investigated the influence of metallic environments on the performance of antennas. Besides the metal environment, also the animal's body will affect the antenna design and its optimization [13] [14]. To obtain accurate link budgets, one needs to model the antennas and the channel jointly, which is not available for the considered use case. For this paper, we aim to use $868 \mathrm{MHz}$. This opens the possibility to use radio technologies like LoRaWAN, Sixfox and other low-power IoT technologies. For this, the channel of the configuration will be characterized and a link budget will be calculated based on simulations.

To the best of the authors' knowledge, this is the first time off-body horse hoof channel is been studied. The novelty of this paper is the antenna-channel design in the unique environment of animal tissue of the horse leg combined with the metallic horseshoe surrounding the antenna. Also the influence of the presence of the ground will be investigated on the propagation and the antenna. 


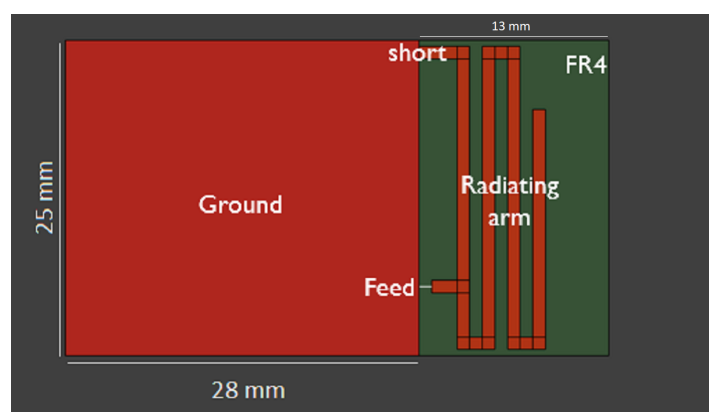

Fig. 1. The layout of the antenna

\section{Method}

In this section, the general approach will be discussed. First an antenna operating in the hoof pad is designed while enclosed with the horse leg and the horseshoe. The parameters of interest are the return loss, bandwidth (BW), center frequency and total efficiency of the antenna. The BW is here defined as the range of frequencies which have a $\left|S_{11}\right|$ below $-10 \mathrm{~dB}$. In this step, the influence of the electrical properties of the horse leg on the antenna will be studied.

\section{A. Antenna Design}

The antenna design has to satisfy the spacial constrains of the device. In this work, the assumption is that the device will be installed in a large hoof pad with dimensions ca. $150 \times$ $150 \times 4 \mathrm{~mm}^{3}$. A border of $2 \mathrm{~cm}$ has to be kept clear. Here the iron will press the pad against the hoof.

1) Antenna: A planar inverted F-antenna is chosen as the antenna for the device. Fig. 1 shows the antenna lay-out. The target center frequency is $868 \mathrm{MHz}$ (BW should be wide enough for LoRa, $250 \mathrm{kHz}$ ). The design is based on the works of Lizzi et al. [15]. The structure is easy to adapt. The placement of the feed can be used to match the antenna and the length of the arm determines the center frequency. With a well understood antenna, the examination of the channel can be performed. Another advantage of this antenna are the volumetric properties. They fulfill the current constrains of the device. The dimensions of the antenna are $43 \times 25 \times 1$ $\mathrm{mm}^{3}$. The antenna itself is $15 \mathrm{~mm}$ long. The dielectric is FR-4 $\left(\varepsilon_{r}=4, \sigma=0.0023 \mathrm{~S} / \mathrm{m}\right)$. The metal tracks and the ground are perfect electrical conductors (PEC). The antenna is embedded in the pad $\left(\varepsilon_{r}=2.5, \sigma=0 \mathrm{~S} / \mathrm{m}\right)$ (Fig. 2a). This encapsulation will increase the robustness of the parameters of the antenna to the influence of the horse leg and the ground.

2) The configuration: Fig. $2 \mathrm{~b}$ shows the complete configuration. The $20 \mathrm{~cm}$ long leg and the $7 \mathrm{~mm}$ thick horseshoe sandwiches the hoof pad wherein the device lays. The leg made out of bone and muscle (homogeneous both times) have been considered with parameters from human tissue [6]. Another simulation will be done with the horse leg out of muscle. The horseshoe is made out of PEC. This is the main configuration that is examined.

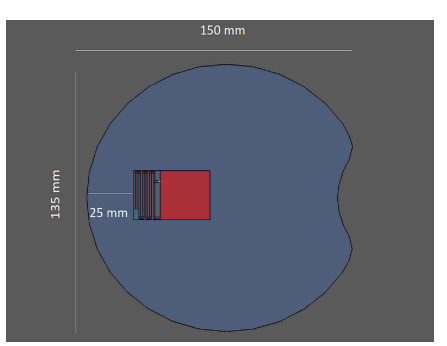

(a) Antenna in the pad

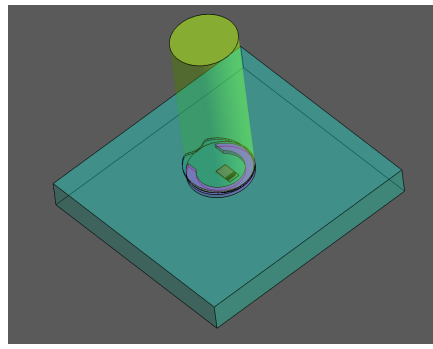

(c) Leg on the ground

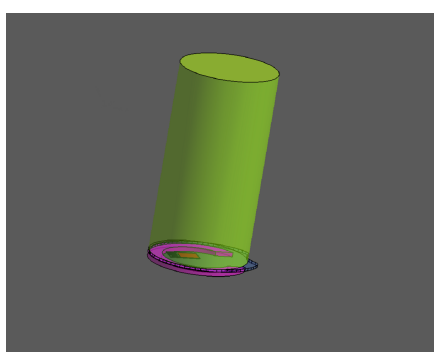

(b) total setup

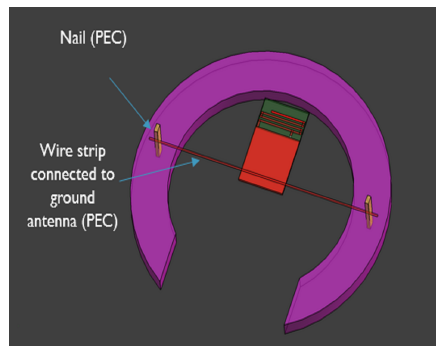

(d) device connected to horseshoe
Fig. 2. The total configuration and the alternative configuration

\section{B. Connecting the Iron Shoe}

In this configuration, the ground of the device is connected to the horseshoe with a wire through the nails. Fig. $2 \mathrm{~d}$ shows the configuration. The effect of the addition of this connection will be examined.

\section{Influence of the Soil}

An interesting situation to examine is the performance of the antenna when the horse leg rests on the ground (Fig. 2c. Here, the underground is taken as dry sand $\left(\varepsilon_{r}=2, \sigma=10\right.$ $\mathrm{S} / \mathrm{m}$ ) with the dimensions of $500 \times 500 \times 50 \mathrm{~mm}^{3}$. The type of soil can very a lot, going from air to wet muddy dirt. When approaching pure water, an effective relative permittivity of 90 can be reached [16], which could substantially influence the properties of the device. These extreme cases will not be examined here.

\section{Path Loss Model and Link Budget}

1) Path Loss model: To calculate the loss of the power as a function of the distance, a path loss model is proposed [10] [17]. The received power $P_{r}(d)$ after a distance $\mathrm{d}$ is given by

$$
P_{r}(d)=\frac{P_{t} G_{t} G_{r} H_{t}^{2} H_{r}^{2}}{d^{4} L}
$$

with $P_{t}$ the power of the transmitter (Tx), $G_{t / r}$ the gain of the receiver $(\mathrm{Rx}) / \mathrm{Tx}, H_{t / r}$ the height w.r.t. the ground of $\mathrm{Rx} / \mathrm{Tx}, \mathrm{d}$ the distance between $\mathrm{Rx}$ and $\mathrm{Tx}$ and $\mathrm{L}$ the losses not accounted for in this path loss model. In this paper, these losses are shadowing losses $\left(M_{s}\right)$ and multi-path fading $\left(M_{f}\right)$. In conclusion, the maximum range (in $\mathrm{dB}$ ) is given by

$$
d_{\max }=\frac{S-P_{t}-G_{r}-G_{t}-M_{f}-M_{s}+2 H_{t}+2 H_{r}}{4}
$$




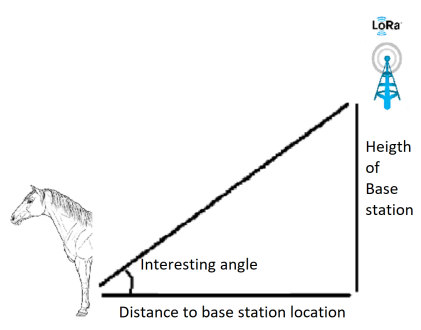

(a) Clarification interesting direction

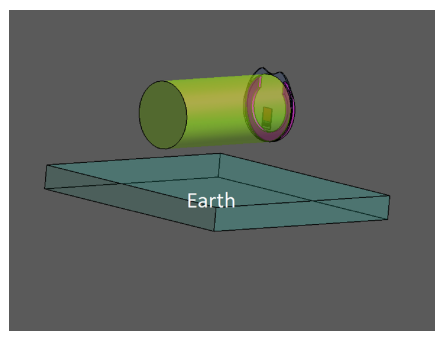

(b) Different orientation w.r.t. the earth
Fig. 3. Clearification link budget calculations

TABLE I

DATARATE TABLE LORA AT A BW OF $125 \mathrm{KHz}$

\begin{tabular}{ccc}
\hline Spreading factor & SNR limit $(\mathrm{dB})$ & Bitrate $(\mathrm{bps})$ \\
\hline 7 & -7.5 & 5469 \\
8 & -10 & 3125 \\
9 & -12.5 & 1758 \\
10 & -15 & 977 \\
11 & -17.5 & 537 \\
12 & -20 & 293 \\
\hline
\end{tabular}

with $\mathrm{S}$ the sensitivity of the considered radio frequency (RF) technology.

2) RF Technologies: Here, the LoRaWAN technology [18] will be examined. The sensitivity of the chip is inversely correlated with the data throughput. LoRa link budget values are listed in Table I for a bandwidth of $125 \mathrm{MHz}$.

The spreading factor SF corresponds with the length in time of a symbol [18]. The sensitivity $\mathrm{S}$ is than calculated as

$$
S=-174+10 \log _{10}(B W)+N F+S N R
$$

with BW the bandwidth in $\mathrm{kHz}$, NF the noise factor which is dependent on the hardware, and SNR the signal to noise ratio. The link budget also depends on the power of the transmitter. In Belgium, the maximum allowed power is $25 \mathrm{~mW}$ or 14 $\mathrm{dBm}$ in the $868 \mathrm{MHz}$ ISM band [18].

3) Link budget: The link budget will be calculated for four configurations: once for the horse leg in the air, once for the leg on the soil, once with the connected device in the air, and once with the connected device on the ground. The range in both cases will be calculated for the maximum gain and once for a interesting direction (Fig. 3a). As interesting direction, a slice of the gain at $5^{\circ}$ from the plane earth is chosen. A trigonometry calculation shows that the angle will be around this value for longer distances $(>100 \mathrm{~m})$ from the base station. From the slice, the maximum gain will be taken. The orientation of the antenna w.r.t. the earth will be different for hoof on ground case and the hoof in air case (shown in Fig. 3b), as a horse turns its hoof $90^{\circ}$ when it lifts it in the air.

\section{E. FDTD simulations}

The calculations of the parameters of interest will be done by Sim4Life, a finite-difference time-domain (FDTD) simulator [19]. For the calculation of the BW and center frequency, a Gaussian signal was excited over the edge source at the feed strip. The center of the Gaussian was $800 \mathrm{MHz}$ and the BW was $250 \mathrm{MHz}$. To simulated an infinite flat earth, the soil was brought into the perfectly match layer for the hoof on the ground configuration. The simulation were then performed with a harmonic excitation of $868 \mathrm{MHz}$ to calculate the gain pattern, efficiency and the $\left|S_{11}\right|$. The number of cells of the grid were $440912 \mathrm{kCells}$ and $668222 \mathrm{kCells}$ for the BW simulations without or with ground, respectively. 472.004 $\mathrm{kCells}$ and $630.808 \mathrm{kCels}$ for the gain pattern simulations without or with ground, respectively.

\section{RESULTS}

\section{A. Configuration 1: The Hoof in the Air}

When the leg is in the air, the antenna has a center frequency of $858 \mathrm{MHz}$ and a bandwidth of $99.5 \mathrm{MHz}$ (only $250 \mathrm{kHz}$ is needed for LoRa), as shown in Fig. 4. At $868 \mathrm{MHz},\left|S_{11}\right|=$ $-19.57 \mathrm{~dB}$. The maximum gain is $-10.0 \mathrm{dBi}$. The gain pattern is shown in Fig. 5a. The gain in the interesting direction is $-11.0 \mathrm{dBi}$. The total efficiency is $3.12 \%$. The low efficiency is due to the absorption of the leg, hence a diminishing of the radiation efficiency, but is satisfying for WBAN's where often the efficiency is $<1 \%$ are obtained. The antenna in free space without horseshoe and horse leg has a radiation efficiency of $50 \%$.

Varying the electric properties of the leg (muscle instead of bones as above) changes the characteristics of the antenna. The center frequency with a muscle phantom changes to $784 \mathrm{MHz}$ with a bandwidth of $99.8 \mathrm{MHz}$, as shown in Fig. 4. This does not satisfy the requirement of Section II anymore. This shows that a good knowledge of the horse leg is needed to design the device. The device should also be robust for different kind of horses and different horses in general. For this, a study of the electrical properties of the horse's hoof area and its variations will be conducted in the future.

\section{B. Configuration 2: Connecting with the Iron}

When the device is connected to the horseshoe via a wire and nails, the center frequency shift of $8 \mathrm{MHz}$ to $886 \mathrm{MHz}$. The bandwidth decreases to $81 \mathrm{MHz}$. This is shown in Fig. 4 . At $868 \mathrm{MHz},\left|S_{11}\right|=-12 \mathrm{~dB}$. The total efficiency, $3.08 \%$, stays the same despite a lower matching. This shows that connecting to the horseshoe results in a slight gain in radiated efficiency. The maximum gain is $-9.99 \mathrm{dBi}$. The maximum gain in the interesting direction of $5^{\circ}$ is $-13.7 \mathrm{dBi}$. The gain pattern is shown in Fig. 5c. Connecting the shoe also changes the shape of the gain pattern.

\section{Configuration 3: Influence of the soil}

When the leg rests on the soil, the center frequency stays roughly the same at $862 \mathrm{MHz}$. The $4 \mathrm{MHz}$ deviation shows the robustness due to the encapsulation in the pad (Section II-A). The bandwidth decreases to $70.0 \mathrm{MHz}$. At $868 \mathrm{MHz}$, $\left|S_{11}\right|=-18.73 \mathrm{~dB}$ and has a maximum gain of $-10.0 \mathrm{dBi}$. The gain in the interesting direction of $5^{\circ}$ is $-15.8 \mathrm{dBi}$. The gain pattern is shown in Fig. 5e. As expected, most of the 


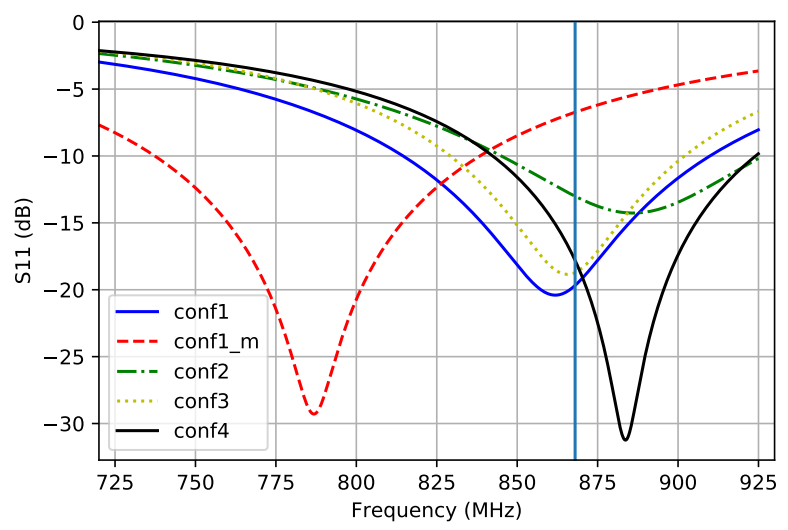

Fig. 4. $S_{11}$ of the different configurations: $\operatorname{conf} 1=$ horse leg without ground, conf1_m = conf 1 with muscle properties, $\operatorname{conf} 2=$ the connected device with the horseshoe without ground, $\operatorname{conf} 3=\operatorname{conf} 1$ with the ground, $\operatorname{conf} 4=\operatorname{conf} 2$ with the ground, the blue vertical line notes the targeted frequency of 868 $\mathrm{MHz}$

power is radiated in the upper half of the space. The soil absorbs the radiation, which explains the lower efficiency of $1.6 \%$, but does not alter the antenna characteristics.

\section{Configuration 4: Connected device on the ground}

As final configuration, the device is now connected to the horseshoe and the hoof is put on the ground. The center frequency shifts $2 \mathrm{MHz}$ to $884 \mathrm{MHz}$ and the BW stays the same at $82 \mathrm{MHz}$. At $868 \mathrm{MHz}$, the $\left|S_{11}\right|=-17.8 \mathrm{~dB}$ (Fig. 4). The total efficiency is $3.3 \%$. The maximum gain is -6.3 $\mathrm{dBi}$ and $-12.7 \mathrm{dBi}$ at $5^{\circ}$ (Fig. $5 \mathrm{~g}$ and $5 \mathrm{~h}$ ). We conclude that it is interesting to connect the device with the horseshoe when the hoof is on the ground.

\section{E. Range per configuration}

We calculated the gain in sections III-A to III-D. The range of the device can be calculated with these results. As mentioned, the maximum allowed radiated power is $14 \mathrm{dBm}$. As base station antenna, a dipole is considered, this has a gain of $2.15 \mathrm{dBi}$. The maximum data rate is from Table I with a bandwidth of $125 \mathrm{kHz}$ is 5469 bps. This results in a sensitivity of $-120 \mathrm{dBm}$. Table II links the link budget calculations with the effective hoof channel. When the hoof is in the air, we estimate that the antenna will be $10 \mathrm{~cm}$ off the ground. Otherwise, it will be $7 \mathrm{~mm}$ off the ground, the thickness of the horseshoe iron. The other path losses are taken from [20]. The configurations are hoof in the air, hoof in the air with the horseshoe connected, hoof on the ground with normal setup and hoof on the ground with connected setup respectively. The calculations show that it is better to send the data when the hoof is in the air $(>1300 \mathrm{~m}$ when in the air compared to $<200 \mathrm{~m}$ when on the ground). A difference of $41 \mathrm{~dB}$ between in the air and on the ground is due to the path loss model, this model punishes low-to-the-ground situated antennas. Yet, it is interesting to see that connecting the device with the horseshoe increases the range with more

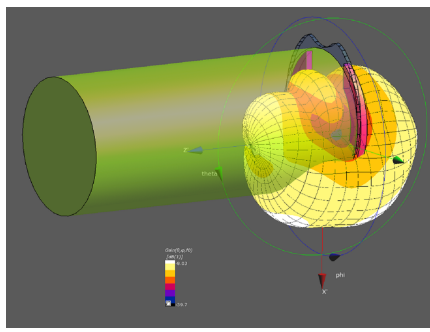

(a) Hoof in the air (earth below)
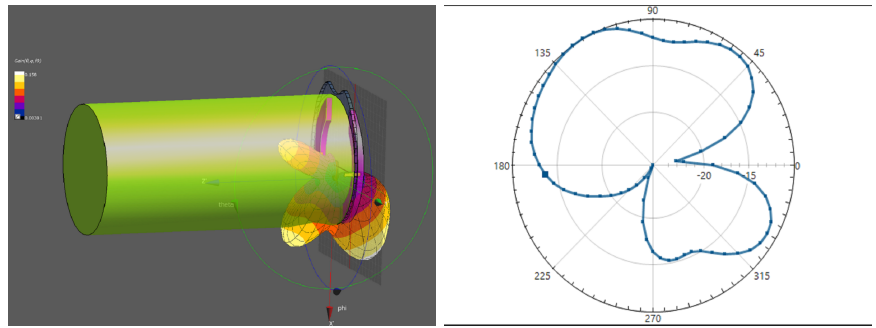

(c) Connected to horseshoe (earth be- (d) Interesting direction (earth above) low)

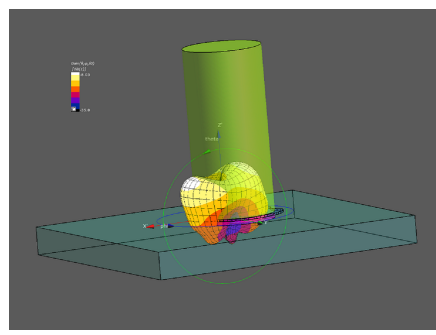

(e) with ground

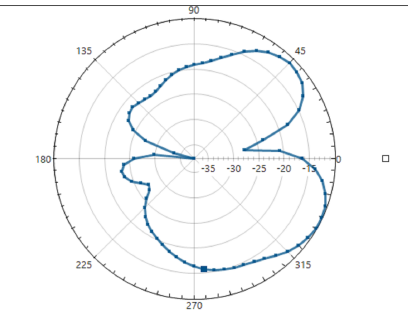

(f) Interesting direction (earth left)

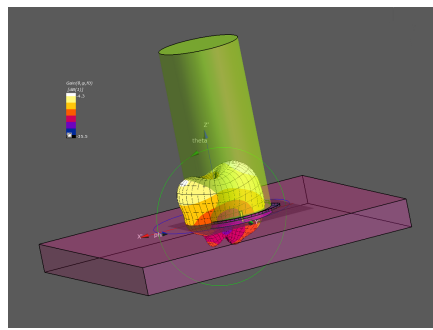

(g) with ground and connected

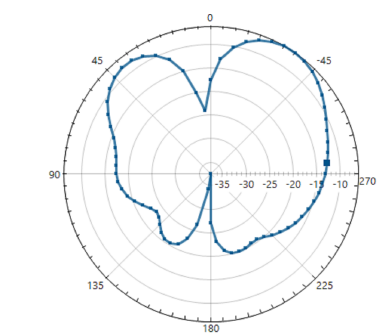

(h) Interesting direction (earth down)

Fig. 5. Gain patterns

than $20 \%$ when the hoof is on the ground. Relative long ranges with relative large data throughput can be obtained when the hoof is in the air.

\section{CONCLUSION}

In this paper, the characterization of the horse hoof environment for sub-gigahertz communication with an inverted-F antenna is investigated using FDTD simulations. The dielectric properties of the hoof have to be understood to design a robust antenna. Connecting the horseshoe with the device can be beneficial to the performance, it can gain up to $20 \%$ in 
TABLE II

RANGE ESTIMATIONS FOR BOTH CONFIGURATIONS

\begin{tabular}{ccccc}
\hline @ $868 \mathrm{MHz}$ & Conf. 1 & Conf. 2 & Conf. 3 & Conf. 4 \\
\hline$P_{t}$ & $14 \mathrm{dBm}$ & $14 \mathrm{dBm}$ & $14 \mathrm{dBm}$ & $14 \mathrm{dBm}$ \\
$G_{r}$ & $2.15 \mathrm{dBi}$ & $2.15 \mathrm{dBi}$ & $2.15 \mathrm{dBi}$ & $2.15 \mathrm{dBi}$ \\
$G_{t}$ & $-10.0 \mathrm{dBi}$ & $-9.99 \mathrm{dBi}$ & $-10.0 \mathrm{dBi}$ & $-6.3 \mathrm{dBi}$ \\
$G_{t}$ at $5^{\circ}$ & $-11.0 \mathrm{dBi}$ & $-13.7 \mathrm{dBi}$ & $-15.8 \mathrm{dBi}$ & $-12.7 \mathrm{dBi}$ \\
$\mathrm{S}$ & $-120 \mathrm{dBm}$ & $-120 \mathrm{dBm}$ & $-120 \mathrm{dBm}$ & $-120 \mathrm{dBm}$ \\
\hline LB & 126 & 126 & 126 & 130 \\
\hline LB at $5^{\circ}$ & 125 & 122 & 120 & 123 \\
\hline \hline$H_{r}$ & $6.9 \mathrm{~dB}$ & $6.9 \mathrm{~dB}$ & $6.9 \mathrm{~dB}$ & $6.9 \mathrm{~dB}$ \\
$H_{t}$ & $-1 \mathrm{~dB}$ & $-1 \mathrm{~dB}$ & $-21.5 \mathrm{~dB}$ & $-21.5 \mathrm{~dB}$ \\
$M_{s}$ & 4 & 4 & 4 & 4 \\
$M_{f}$ & 4.3 & 4.3 & 4.3 & 4.3 \\
\hline PL & $4 \mathrm{~d}-3.5 \mathrm{~dB}$ & $4 \mathrm{~d}-3.5 \mathrm{~dB}$ & $4 \mathrm{~d}+37.5 \mathrm{~dB}$ & $4 \mathrm{~d}+37.5 \mathrm{~dB}$ \\
\hline \hline Range & $1720 \mathrm{~m}$ & $1720 \mathrm{~m}$ & $163 \mathrm{~m}$ & $205 \mathrm{~m}$ \\
\hline Range $5^{\circ}$ & $1631 \mathrm{~m}$ & $1372 \mathrm{~m}$ & $115 \mathrm{~m}$ & $137 \mathrm{~m}$ \\
\hline
\end{tabular}

range. The best way for a efficient communication is sending data when the hoof is in the air. Future work will consist of improving models by constructing a live like horse model. Characterization of the horse hoof will aid the designer to make a robust device for every targeted horse. In later work, the antenna should be converted to dual-band to allow 2.45 $\mathrm{GHz}$ communication. Also the influence of different types of soil is part of future work. Lastly, manufacturing the antenna will validate the results. The preliminary experimental validation will be presented at the conference.

\section{ACKNOWLEDGMENT}

This work was executed within the imec.icon project HoofMATE, a research project bringing together academic researchers and industry partners. The Hoof-MATE project was co-financed by imec and received project support from Flanders Innovation \& Entrepreneurship (project nr. HBC.2018.0536).

\section{REFERENCES}

[1] "Smart Halter I Alerts Equine Distress, Foaling, Colic Signs of Horse," NIGHTWATCH. [Online]. Available: https://www.smarthalter.com/. [Accessed: 17-Oct-2019].

[2] "EquiFone Foaling Alarm." [Online]. Available: http://www.foalingalarm.com/equifone.htm. [Accessed: 17-Oct-2019].

[3] C. A. Balanis, Antenna theory: analysis and design. New York: Harper \& Row, 1982.

[4] S.J. Ambroziak, "Impact of radio wave polarisation on off-body communications in indoor environments," in $9^{\text {th }}$ European Conference on Antennas and Propagation (EuCAP), Portugal, pp. 1 - 3, 2015.

[5] S. van Roy et al., "Dynamic Channel Modeling for Multi-Sensor Body Area Networks," IEEE Trans. Antennas Propagat., vol. 61, no. 4, pp. 2200-2208, Apr. 2013.

[6] S. Gabriel et al., "The dielectric properties of biological tissues: III. Parametric models for the dielectric spectrum of tissues," Physics in Medicine and Biology,vol. 41, pp. 2271-2293, 1996.

[7] K. H. Kwong et al., "Wireless Sensor Networks in Agriculture: Cattle Monitoring for Farming Industries," PIERS Online, vol. 5, no. 1, pp. 31-35, 2009.

[8] K. H. Kwong et al., "Practical considerations for wireless sensor networks in cattle monitoring applications," Computers and Electronics in Agriculture, vol. 81, pp. 33-44, Feb. 2012.

[9] S. Benaissa et al., "Experimental characterisation of the off-body wireless channel at $2.4 \mathrm{GHz}$ for dairy cows in barns and pastures," Computers and Electronics in Agriculture, vol. 127, pp. 593-605, Sep. 2016.
[10] S. Benaissa et al., "Internet of animals: characterisation of LoRa sub$\mathrm{GHz}$ off-body wireless channel in dairy barns," Electronics Letters, vol. 53, no. 18, pp. 1281-1283, Aug. 2017.

[11] E. Reusens et al., "Characterization of On-Body Communication Channel and Energy Efficient Topology Design for Wireless Body Area Networks," IEEE Trans. Inform. Technol. Biomed., vol. 13, no. 6, pp. 933-945, Nov. 2009.

[12] E. Tanghe, W. Joseph, P. Ruckebusch, L. Martens, and I. Moerman, "Intra-, Inter-, and Extra-Container Path Loss for Shipping Container Monitoring Systems," Antennas Wirel. Propag. Lett., vol. 11, pp. 889-892, 2012.

[13] D. Nikolayev, W. Joseph, M. Zhadobov, R. Sauleau, and L. Martens, "Optimal radiation of body-implanted capsules",Phys. Rev. Lett., vol. 122, no. 10, p. 108101, Mar. 2019.

[14] D. Nikolayev, M. Zhadobov, and R. Sauleau, "Impact of tissue electromagnetic properties on radiation performance of in-body antennas", IEEE Antenn. Wireless Propag. Lett., vol. 17, no. 8, pp. 1440-1444, Aug. 2018.

[15] L. Lizzi, F. Ferrero, C. Danchesi, and S. Boudaud, "Miniature Multiband Inverted-F Antenna over an Electrically Small Ground Plane for Compact IoT Terminals," Wireless Communications and Mobile Computing, vol. 2018, pp. 1-8, Jul. 2018.

[16] P. O. Risman and B. Wäppling-Raaholt, "Retro-modelling of a dual resonant applicator and accurate dielectric properties of liquid water from $-20{ }^{\circ} \mathrm{C}$ to $+100{ }^{\circ} \mathrm{C}, "$ Meas. Sci. Technol., vol. 18, no. 4, pp. 959-966, Apr. 2007.

[17] I. Glover and P. M. Grant, Digital communications, 3rd ed. Harlow, England; New York: Prentice Hall, 2010.

[18] "LoRaWAN," The Things Network, 16-Oct-2019. [Online]. Available: https://www.thethingsnetwork.org/docs/lorawan/. [Accessed: 17Oct-2019].

[19] zurich med tech. [Online]. Available: https://zmt.swiss/. "zurich med tech,” Sim4Life. [Online]. Available: https://zmt.swiss/. [Accessed: 17Oct-2019].

[20] S. Benaissa, "LoRa in-to-out-body link budget for animals," unpublished 